\title{
Cinquante années de recherches sur les débuts de l'Aurignacien en Europe occidentale
}

\author{
FRANÇOIS DJINDJIAN ${ }^{1}$
}

RESUME

Une histoire des cinquante dernières années de recherches sur les débuts de l'Aurignacien est tentée ici. Cet historique retrace brièvement les débuts entre 1860 et 1950 de la connaissance de l'Aurignacien resituant dans leur contexte les apports respectifs de Lartet, De Mortillet, Breuil et Peyrony. Puis, les résultats des recherches des années 1950

à 1990 des différents acteurs (D. de Sonneville-Bordes, F. Bordes, H. M. Movius, H. Delporte, G. Laplace, J. Hahn, N. Soler, F. Bazile, F. Champagne, etc.) concernant les débuts de l'Aurignacien sont analysés à la lueur des nouvelles données de fouilles en Aquitaine (Roc de

Combe, La Ferrassie, Le Facteur, Caminade, Le Flageolet I, Le Piage), en Pyrénées (Gatzarria, Cueva Morin), en Jura-Souabe (Geissenklosterle) et sur la côte méditerranéenne (abri Mochi, La Laouza, l'Arbreda, L'Esquicho-Grapaou). Les apports des recherches sur le paléoenvironnement pour la reconstitution du climat et des méthodes mathématiques et informatiques dans les années 70 à la structuration chronologique de l'Aurignacien sont développés. Les

\section{RESUMEN}

En este trabajo se realiza una tentativa a la historia de las investigaciones sobre los inicios del Auriñaciense en los últimos cincuenta años. En esta historia se tratan brevemente los inicios del conocimiento del Auriñaciense entre 1860 y 1950 con las aportaciones de Lartet, de Mortillet, Breuil y Peyrony. Después, se analizan los resultados de las investigaciones entre 1950 y 1990, llevadas a cabo por diferentes autores (D. De SonnevilleBordes, F. Bordes, H. M. Movius, $H$. Delporte, G. Laplace, J. Hahn, N. Soler, F. Bazile, F. Champagne, etc.) a la luz de los nuevos datos obtenidos de excavaciones antiguas en Aquitania (Roc de Combe, La Ferrassie, Le Facteur, Caminade, Le Flageolet I, Le Piage), en los Pirineos (Gatzarria, Cueva Morin), en Jura-Suabia (Geissenklosterle) y la costa mediterránea (abri Mochi, La Laouza, l'Arbreda, L'Esquicho-Grapaou). Se trata también la aportación realizada por las investigaciones consagradas a la reconstrucción del paleoclima y a los métodos matemáticos e informáticos de los años 70 con los que se desarrolla el marco cronológico del Auriñaciense.

1 Université de Paris 1 et CNRS UMR 7041. 
différentes données à l'origine de l'existence d'un Protoaurignacien, d'un Aurignacien 0 en Périgord, d'un Aurignacien initial et la question de linterstratification entre Castelperronien et Aurignacien sont discutées. Les récentes critiques depuis le début des années 90 concernant la fiabilité du cadre paléoclimatique des remplissages d'abrissous-roche et la pertinence des approches typologiques sont examinées. Les conclusions amènent l'auteur à proposer suggérer que les débuts de l'Aurignacien, encore mal connus, ne sont pas uniformes

suivant les régions et dans le temps.

En Europe occidentale, l'existence d'un Aurignacien initial semble prouvé sur la côte méditerranéenne de la Ligurie jusqu'en Catalogne. L'expansion aurignacienne suit alors la bordure septentrionale pyrénéenne de l'Aude jusqu'en Cantabres et en Asturies. Puis, sous sa forme Aurignacien I, il remonte vers le Nord et occupe toute l'Aquitaine pendant le premier épisode froid du denier pléniglaciaire.

\section{MOTES CLÉFS}

Aurignacien, Historiographie, Sud-Ouest de Europe.
También se discutirán los diferentes datos entorno a el origen de la existencia de un Protoauriñaciense, de un Auriñaciense 0 en Perigord, de una Auriñaciense inicial y sobre la cuestión de la interestratificación entre el Chatelperroniense y el Auriñaciense, asi como las recientes críticas de los años 90 sobre la fiabilidad del cuadro paleoclimático a partir de las estratigrafías de los abrigos y de las aproximaciones tipológicas. Las conclusiones llevan a sugerir al autor que los origenes del Auriñaciense, aún mal conocido, no es uniforme en el tiempo y el espacio. En Europa occidental, la existencia de un Auriñaciense inicial queda probada en la costa mediterránea desde la Liguria hasta Cataluña. También su expansión por la vertiente norte de los Pirineos desde el Aude hasta Cantabria y Asturias. Después, en e su forma de Auriñaciense I, remonta hacia el norte $y$ ocupa toda la Aquitania durante el primer episodio frío del último pleniglaciar.

\section{PALABRAS CLAVE}

Auriñaciense, Historiografía, Suroeste de Europa.

\section{INTRODUCTION: LES DEBUTS DES RECHERCHES SUR L'AURIGNACIEN (1861-1948)}

II n'est pas inutile de revenir même brièvement sur la découverte et l'individualisation de l'Aurignacien dans la préhistoire de la seconde moitié du $X I X^{\circ}$ siècle. L'Aurignacien, découvert et fouillé par Lartet en 1861 dans le site d'Aurignac en Haute-Garonne, a été enterré par le positiviste De Mortillet, qui, acquis aux idées évolutionnistes de Darwin, a cherché dans l'évolution typologique des outils en silex taillé paléolithiques les mêmes lois que celle des évolutions morphologiques des espèces fossiles. C'est donc à Henri Breuil, à la suite d'une bataille stratigraphique homérique qui a eu son épilogue au congrès de Genève en 1911 (Breuil, 1912), qu'est revenu l'honneur d'être le second géniteur, après Lartet, de l'Aurignacien. L'Aurignacien d'Henri Breuil, comme chacun sait, est subdivisé en Aurignacien inférieur (appelé actuellement Castelperronien ou Châtelperronien), Aurignacien moyen (c'est l'actuel Aurignacien stricto sensu) et Aurignacien supérieur (appelé actuellement Gravettien sur la proposition de D. Garrod (1938)). 
Entre les deux guerres, les propres fouilles de D. Peyrony au grand abri de La Ferrassie (Peyrony, 1934) et à l'abri de Laugerie-Haute (Peyrony D. et E., 1938), et les résultats des fouilles de Lacorre à La Gravette (Lacorre, 1960) vont en effet fournir à $D$. Peyrony plusieurs séquences de référence pour élaborer un modèle nouveau et plus complexe pour l'Aurignacien de Breuil (Peyrony, 1933). Deux évolutions parallèles sont proposées, le Périgordien et l'Aurignacien, sur la base de différences typologiques entre la présence de pièces à retouche abrupte (pointes de Châtelperron, pointes de la Gravette) pour le premier et de grattoirs et burins épais à retouche lamellaire (grattoir caréné, grattoir à museau, burin busqué) pour le second. L'Aurignacien est subdivisé en cinq faciès chronologiques sur la base de l'industrie osseuse des sagaies: I à IV, correspondant aux niveaux de la séquence aurignacienne de La Ferrassie et $\mathrm{V}$ pour le niveau de Laugerie-Haute situé audessus du Gravettien. Le Périgordien comprend également cinq faciès chronologiques: le Périgordien I (Castelperronien), le Périgordien II (le niveau E' de La Ferrassie, dont nous reparlerons plus loin), le Périgordien III (le niveau B de Laugerie-Haute-Ouest), le Périgordien IV (présent à La Gravette) et enfin le Périgordien $V$ (subdivisé en trois sous-faciès sur la base des niveaux gravettiens $\mathrm{J}$, $\mathrm{K}, \mathrm{L}$ de La Ferrassie: $\mathrm{Va}$, à pointes de la Font-Robert; $\mathrm{Vb}$ à éléments tronqués; $\mathrm{Vc}$ à burins de Noailles). Plus tard, D. Peyrony (1948) complexifiera encore ce modèle pour y inclure les industries atypiques de Corrèze.

\section{CONTINUATION ET FIN DU MODELE PERIGORDIEN DE D. PEYRONY (1950-1990)}

La politique scientifique de l'Institut du Quaternaire à Bordeaux, suite à l'arrivée de $F$. Bordes et de $D$. de Sonneville-Bordes, est de prolonger le modèle périgordien de $D$. Peyrony, qui ne sera abandonné que dans les années 90 . Dans les années 50 , partout en France, les chercheurs essaient de généraliser le modèle périgordien bien au-delà du Périgord. J. Combier (1951) attribue une petite série de surface de Roclaine dans le bassin de la Saône au Périgordien II. M. Escalon de Fonton (1956) à la Salpétrière propose une interstratification de niveaux aurignaciens et périgordiens en se basant sur des petites séries de pièces de débitage en fait indéterminables (Bazile, communication orale et Djindjian et alii, 1999, p. 342 pour une révision de la séquence). L. Pradel publie le site de Laraux (Pradel et Chollet, 1950) avec une séquence complète du Périgordien $V$, en «inventant" une lentille avec quelques pointes de la Font-Robert. II réitère sur le site des Cottés (Pradel, 1959) en inventant la pointe des Cottés, un pseudo-intermédiaire typologique entre les pointes de Châtelperron à dos courbe et les pointes de la Gravette à dos droit, dans un soi-disant Périgordien II (couche G). Ces excès de zèle n'empêchent pas l'Institut du Quaternaire d'adapter le modèle périgordien en Périgord en fonction des données des nouvelles fouilles ou de la révision des anciennes collections. Le point le plus faible de la construction de Peyrony, que les chercheurs ne découvriront que très progressivement, est d'être basé sur une construction plus 
typologique que stratigraphique. Très vite, l'existence du Périgordien moyen (Périgordien II et III), contemporain de l'Aurignacien I à IV, est mise en doute. Les fouilles de H. M. Movius à l'abri Pataud aux Eyzies, commencées dans les années 50 , révèlent que le Périgordien III (alias la couche $B$ de Laugerie-Haute Ouest) est en fait postérieur au Périgordien $V$ et devient dès lors Périgordien VI. Les travaux de $G$. Laplace sur le Protoaurignacien amènent $D$. de Sonneville-Bordes (Sonneville-Bordes, 1955) à réviser l'industrie de la couche E' de La Ferrassie (la petite série possède des lamelles Dufour) et de lui retirer le statut de référence éponyme du Périgordien II, qui se retrouve alors sans représentants officiels.

H. Delporte (1953) et A. Leroi-Gourhan (1961), les plus actifs à refuser le modèle périgordien, proposent un schéma différent: le Périgordien I, nommé Castelperronien ou Châtelperronien du nom du site éponyme de Châtelperron, situé dans le bassin de la Loire (Allier), (où $\mathrm{H}$. Delporte a effectué quelques fouilles sans grands résultats dans les années 1951-54 (Delporte, 1957)), doit être vu comme une évolution ultime des industries du Paléolithique moyen, sous l'effet d'une acculturation ou d'une évolution au début du Paléolithique supérieur, comme semblent le montrer les données des fouilles de la grotte du Renne à Arcy-surCure. La découverte, dans les années 70, à Saint-Césaire (Charente-Maritime), d'un squelette néandertalien dans un niveau castelperronien, dont l'attribution stratigraphique a été mise en doute au moment de sa découverte par F. Bordes, devait contribuer fortement à confirmer ce modèle admis depuis (Levèque, 1987).

H. Delporte et A. Leroi-Gourhan proposent également que les Périgordiens IV et $\mathrm{V}$, devenus après la disparition du Périgordien II et III, le Périgordien supérieur, soient nommés Gravettien, faisant du Périgord un point d'arrivée des peuplements gravettiens venus d'Europe centrale via le nord de la France et non son épicentre (Djindjian et alii, 1999).

L'étape suivante du démantèlement du modèle périgordien fut la question de l'Aurignacien $V$. Le niveau éponyme de cette industrie est la couche $D$ de LaugerieHaute Ouest, découverte par D. Peyrony entre Périgordien III (devenu VI) et Protomagdalénien (une industrie à l'allure gravettienne que D. Peyrony avait nommée Protomagdalénien sur la base d'une sculpture, le bois de renne aux deux mammouths affrontés, dont la facture lui semblait plus proche de l'art du Magdalénien moyen que du Périgordien). Les fouilles de $F$. Bordes modifient l'attribution stratigraphique de cette industrie en la situant cette fois à LaugerieHaute Est entre le Protomagdalénien et le Protosolutréen. Pour F. Bordes, d'ailleurs, le Protomagdalénien (au sens Peyrony) n'est autre qu'un Périgordien final (Bordes et Sonneville-Bordes, 1966), auquel il attribuerait volontiers le nom de Périgordien VII. Or il fut impossible de trouver d'autres industries attribuables à l'Aurignacien V, sinon l'Aurignacien moyen et terminal d'Escalon de Fonton à La Salpétrière (1956), puis les attributions «forcées» de L. Pradel (1952) sur le site du Fontenioux (niveau aurignacien évolué au dessus d'un niveau castelperronien interprété comme un niveau Aurignacien $\mathrm{V}$ au-dessus d'un niveau gravettien) et de 
P. Y. Demars (1985) sur des séries aurignaciennes de surface. II revient à Ch. Leroy-Prost d'avoir découvert, sans oser l'écrire, la vérité sur cette industrie, dans sa thèse sur l'industrie osseuse aurignacienne (Leroy-Prost, 1975, 1978), en constatant que les sagaies de l'Aurignacien $V$ de Laugerie-Haute sont des sagaies sans aucune similitude avec les sagaies aurignaciennes mais tout à fait semblables aux sagaies solutréennes (sagaies à base en biseau simple). Progressivement, D. de Sonneville-Bordes (1982) renonce à l'existence de cette série lithique de l'Aurignacien $\mathrm{V}$, que plusieurs préhistoriens, qui l'ont examinée, soupçonnent d'avoir été triée. Au XXIV ${ }^{\circ}$ Congrès Préhistorique de France, à Carcassonne, en 1994, deux communications proposent indépendamment l'abandon de l'Aurignacien V: Djindjian (1999), en se basant sur la révision des industries de l'Aurignacien terminal de La Salpêtrière, de Laugerie-Haute et de sites espagnols cantabriques et méditerranéens, définit ces niveaux comme une industrie de transition entre Gravettien et Solutréen, avec comme seuls traits aurignacoïdes, l'adoption d'un débitage lamellaire. Zilhao et al. (1999) arrivent à une conclusion identique à partir des fouilles de sauvetage de l'abri Casserole aux Eyzies, de la révision de la série de l'Aurignacien $V$ de Laugerie-Haute qu'ils interprètent comme une série artificiellement constituée par $F$. Bordes à partir des séries du Protosolutréen pour retrouver le niveau de D. Peyrony et de sites portugais à la transition Gravettien-Solutréen. L'Aurignacien $\mathrm{V}$ et la contemporanéité de l'Aurignacien et du Périgordien sont définitivement réfutés. A ce stade de nos connaissances, trois industries se succèdent donc: le Castelperronien, l'Aurignacien et le Gravettien, revenant ainsi, plus de quatre-vingt ans après, à la terminologie près, à la chrono-stratigraphie d' $\mathrm{H}$. Breuil du début du siècle. Le démantèlement final du modèle périgordien revient à Djindjian et Bosselin (1994), qui révisent la chrono-stratigraphie du Périgordien supérieur (Périgordien IV, V, VI). IIs mettent en évidence que la succession des faciès est uniquement liée à une superposition artificielle de deux séquences sans corrélation stratigraphique aucune: la séquence de La Gravette et la séquence de La Ferrassie. La confrontation des données issues de l'analyse des données des séries lithiques, des relations stratigraphiques entre niveaux et la synthèse des datations ${ }^{14} \mathrm{C}$ met en évidence que le Périgordien IV (à pointes de la Gravette) défini à La Gravette et le Périgordien Vb (à pointes de la Gravette et à éléments tronqués) défini à La Ferrassie sont en fait un seul et même faciès et que la succession stratigraphique n'est pas Périgordien $\mathrm{IV}, \mathrm{Va}, \mathrm{Vb}, \mathrm{Vc}, \mathrm{VI}$ mais $\mathrm{Va}$, $I V=V b, V c, V I$ c'est-à-dire Gravettien ancien (ex-Périgordien $V a$ ) à pointes de la Font-Robert, pointes de la Gravette et fléchettes, Gravettien ancien (exPérigordien IV et ex-Périgordien Vb) à pointes de la Gravette seules, Gravettien moyen (ex-Périgordien $\mathrm{Vc}$ ) à burins de Noailles puis à burins du Raysse, Gravettien récent (ex-Périgordien VI) à pointes de la Gravette et microgravettes, et enfin Gravettien final (ex-Protomagdalénien Peyrony). 


\section{G. LAPLACE, LE PROTOAURIGNACIEN ET LE MODELE DU SYNTHETOTYPE}

Si le modèle du synthétotype de G. Laplace est directement inspiré des travaux de A.C. Blanc, lors de son séjour à l'Ecole Française de Rome, et peut rentrer sous l'étiquette de structuralisme marxiste, son application aux débuts du Paléolithique supérieur en Europe occidentale est entièrement de son fait. De ce qui nous intéresse plus particulièrement ici de son œuvre majeure «Recherches sur l'origine et l'évolution des complexes leptolithiques" (Laplace, 1966), plus que le synthétotype, c'est l'identification d'un Protoaurignacien, à partir d'un ensemble, disparate et mélangé pour ses détracteurs, de niveaux aurignaciens caractérisés par l'abondance des lamelles Dufour (lamelles à retouche inverse) trouvés notamment en Pyrénées (Gatzaria, Les Abeilles, Isturitz), en Espagne cantabrique (Cueva Morin, Pendo, Castillo), en Corrèze (Dufour, Font-Yves, Bos del Ser), en Périgord ( $E^{\prime}$ de La Ferrassie), en Italie (abri Mochi, Castelcivita, etc.), en BasseAutriche (Krems).

Mais, à partir de la fin des années 60 , les nouvelles fouilles apportaient plus d'arguments aux détracteurs du Protoaurignacien qu'à ses défenseurs. En Périgord, à la base des séquences aurignaciennes, notamment à l'abri Pataud, à l'abri du Facteur, à l'abri Caminade, à l'abri du Roc de Combe, à l'abri du Flageolet I, au grand abri de La Ferrassie (où $\mathrm{H}$. Delporte ne retrouva pas l'équivalent de la couche E'), aucun niveau à lamelles Dufour ne fut trouvé. Au contraire, des lamelles Dufour furent rencontrées dans des niveaux d'Aurignacien évolué à Roc de Combe (couche 6) et à Caminade-Est (couche D2), comme celles que A. Leroi-Gourhan avait trouvées dans l'Aurignacien de la couche VII de la grotte du Renne à Arcy-sur-Cure (Yonne), couche qu'il avait attribuée à un Aurignacien évolué situé dans l'éponyme interstade d'Arcy. En Italie, les niveaux attribués au Protoaurignacien, furent progressivement datés par le ${ }^{14} \mathrm{C}$ et se révélèrent plus jeunes que prévus et la question d'un peuplement tardif de la péninsule italienne par l'Aurignacien seulement à partir de l'épisode d'Arcy commença à se poser. En Corrèze, les révisions des séries aurignaciennes des sites majeurs conclurent plutôt à l'attribution de ces séries (Dufour, Chanlat, Font-Yves, Bos del Ser) à un Aurignacien évolué qu'à un Protoaurignacien (Sonneville-Bordes, Djindjian, Mazières, Demars). A la fin des années 70 , le Protoaurignacien semblait définitivement remis en cause.

\section{LES FOUILLES DE H. DELPORTE EN PERIGORD: LA ROCHETTE, LE FACTEUR, LA FERRASSIE}

Le premier site étudié par $\mathrm{H}$. Delporte en Périgord fut l'abri de La Rochette (Delporte, 1964), où il découvrit une séquence malheureusement remaniée comprenant du Moustérien, du Castelperronien, de l'Aurignacien et du Gravettien. Puis l'abri du Facteur à Tursac, non loin des Eyzies, lui fournit une séquence 
majeure qu'il fouilla de 1955 à 1960 (Delporte, 1968), avec principalement plusieurs niveaux d'Aurignacien, un niveau de Gravettien à burins de Noailles, et un niveau qualifié par $\mathrm{H}$. Delporte lui-même d'Aurignaco-Périgordien, dont il eut du mal à admettre que c'était un mélange d'Aurignacien et de Gravettien. Des niveaux aurignaciens, on retiendra le niveau 21, Aurignacien I situé dans un épisode froid, le niveau 19, Aurignacien II situé dans l'épisode d'Arcy, le niveau 17, très pauvre, situé dans un épisode froid sans doute Aurignacien III, et enfin le niveau 15, situé dans l'épisode de Maisières, ex-« Aurignaco-Périgordien», sans doute un Aurignacien IV et mélangé avec un Gravettien ancien. Cette séquence, de par certaines différences avec la séquence "officielle" du grand abri de La Ferrassie établie par D. Peyrony, entraîna H. Delporte à ouvrir de nouvelles fouilles au grand abri de La Ferrassie, entre 1968 et 1973, avec l'appui des chercheurs de l'Institut du Quaternaire, à Bordeaux (Delporte, Maziere et Djindjian, 1977; Delporte, 1984). Du grand abri de la Ferrassie, il ne subsistait plus que des lambeaux du sommet de la stratigraphie, et en particulier du Gravettien. Par contre, la séquence aurignacienne, castelperronienne et moustérienne était intacte, mais en deux endroits distincts, une coupe frontale et une coupe sagittale, avec des possibilités limitées de raccord entre les deux coupes. Les fouilles d'H. Delporte furent plus qu'un ravivage de coupes, permettant d'obtenir pour la quasi-totalité des niveaux un nombre suffisant d'outils pour que ces nouvelles fouilles définissent le nouvel ensemble de référence de La Ferrassie, en remplacement de celui de D. Peyrony. $H$. Delporte découvrit en effet non pas, comme D. Peyrony, cinq niveaux aurignaciens $E^{\prime}, F, H, H^{\prime}, H^{\prime \prime}$ et trois niveaux périgordiens $J, K, L$ mais plus d'une vingtaine de niveaux bien différenciés dont l'abondante industrie lui permit de réviser les faciès chronologiques aurignaciens et de confirmer l'intérêt de la séquence pour les débuts du paléolithique supérieur.

$\begin{array}{ll}\text { Gravettien à burins de Noailles } & (\mathrm{B} 3, \mathrm{~B} 4), \\ \text { Gravettien à pointes de la Gravette } & (\mathrm{C} 4, \mathrm{D} 1), \\ \text { Gravettien à pointes de la Font-Robert } & (\mathrm{E} 1, \mathrm{D} 2, \mathrm{D} 3), \\ \text { Aurignacien IV: } & (\mathrm{E} 1, \mathrm{~F}, \mathrm{Gf}, \mathrm{Gsn}, \mathrm{Gss}), \\ \text { Aurignacien III } & (\mathrm{H}, \mathrm{I1}), \\ \text { Aurignacien IIb } & (\mathrm{Js}, \mathrm{Jf}, \mathrm{I2}), \\ \text { Aurignacien IIa } & (\mathrm{K} 4, \mathrm{~K} 3 \mathrm{~b}, \mathrm{~K} 3 \mathrm{a}, \mathrm{K} 2 \mathrm{f}, \mathrm{K} 2 \mathrm{~s}), \\ \text { Aurignacien I: } & (\mathrm{K} 7, \mathrm{~K} 6, \mathrm{~K} 5), \\ \text { Castelperronien } & (\mathrm{M} 1, \mathrm{L3}), \\ \text { Moustérien } & (\mathrm{M} 2)\end{array}$

Avec ces nouvelles données, se posa néanmoins la question de la mise en correspondance de la nouvelle séquence avec l'ancienne séquence établie par $D$. Peyrony. Très vite, $H$. Delporte se rendit compte que la stratigraphie de $D$. Peyrony avec des niveaux bien individualisés séparés par des couches stériles ne correspondait pas à la séquence de ses propres fouilles. C'est que D. Peyrony, comme bien d'autres fouilleurs de son époque, entre les deux guerres, faisait des 
relevés stratigraphiques schématiques et de mémoire, en quelque sorte idéalisés et non des relevés réels. Des traitements statistiques ont permis de comprendre la réalité: une régression multiple (inédite) a permis d' "expliquer» les couches de Peyrony par celles de Delporte: il en résulte que le niveau $F$ est un mélange des niveaux $\mathrm{K} 6, \mathrm{~K} 5, \mathrm{~K} 4$ et $\mathrm{K} 3$ donc d'Aurignacien I et d'Aurignacien II, et ainsi de suite dans la séquence. Les faciès aurignaciens de référence de Peyrony ne sont donc plus valables et seuls ceux plus précis de $\mathrm{H}$. Delporte le sont. On peut en tirer une conséquence importante concernant l'Aurignacien ancien (I): D. de SonnevilleBordes (1960) avait défini deux faciès différents pour l'Aurignacien ancien: le faciès Castanet et le faciès Lartet-Ferrassie. L'analyse approfondie des séries caractérisant le faciès Lartet-Ferrassie montre que comme à La Ferrassie couche $F$, ce sont des mélanges d'un niveau Aurignacien I avec un niveau Aurignacien II sus-jacent. L'Aurignacien I ou Aurignacien ancien n'est donc caractérisé que par un seul et même faciès, le faciès Castanet, dans la définition précise donnée par Delporte (1984) ou Djindjian (1988).

\section{LA QUESTION DE L'AURIGNACIEN O EN PERIGORD (1967-1981)}

Entre le Protoaurignacien de G. Laplace et le modèle périgordien de l'Institut du Quaternaire, H. Delporte était convaincu d'un troisième modèle, un "syncrétisme", basé sur l'existence en Périgord d'un Aurignacien 0, précédant donc l'Aurignacien ancien ou Aurignacien I. Un certain nombre de décomptes d'assemblages typologiques allaient le conforter dans cette opinion. Le premier était la série de la couche E' de La Ferrassie que D. de Sonneville-Bordes, même si elle ne pouvait plus lui reconnaître le statut d'un Périgordien II, ne pouvait cependant pas, en pleine guerre protoaurignacienne, lui attribuer celui de Protoaurignacien, ce que fit $H$. Delporte avec diplomatie en parlant non pas de Protoaurignacien mais d'Aurignacien 0 . D'autres séries à la base de stratigraphies aurignaciennes en Périgord vinrent à la rescousse: la couche 5D de La Rochette et la couche $G$ de Caminade Est ${ }^{2}$.

II fut néanmoins très déçu de ne pas retrouver à La Ferrassie la couche E', au point de vouloir voir à tort dans le maigre niveau $K 7$, qui est identique statistiquement à $\mathrm{K} 6$ et $\mathrm{K} 5$, un niveau plus ancien. La série $\mathrm{E}$ 'à La Ferrassie n'est sans doute qu'un mélange entre des outils castelperroniens et des outils aurignaciens, comme l'avait bien pressenti $\mathrm{D}$. de Sonneville-Bordes, ce qui pose la question de la présence de lamelles à retouche marginale dans le Castelperronien, dont l'étude a été particulièrement négligée jusqu'à présent.

2 Je me souviens encore, en 1974, pendant la soutenance de ma maîtrise qui présentait pour la première fois des plans factoriels d'assemblages typologiques aurignaciens, l'indignation d'H. Delporte, quand j'ai interprété ces assemblages, qui s'étaient réfugiés près de l'origine des axes, comme atypiques ou aberrants: il m'a coupé la parole pour dire qu'ils montraient en fait l'existence d'un faciès original Aurignacien 0 , dont il était persuadé de l'existence et que l'analyse factorielle révélait indiscutablement. 
De nouvelles découvertes allaient cependant abonder en son sens. Au Piage, dans le Lot, F. Champagne et R. Espitalié $(1967,1981)$ publient une stratigraphie avec une interstratification de l'Aurignacien et du Castelperronien. Le niveau aurignacien le plus ancien, $\mathrm{K}$, possède de nombreuses lamelles Dufour. $\mathrm{F}$. Bordes, réagit immédiatement en montrant qu'une interstratification similaire existait dans la séquence de l'abri de Roc de Combe, dans le Lot également (Bordes et Labrot, 1967). Pour F. Bordes, ces découvertes arrivent à point pour relancer le modèle périgordien en démontrant le parallélisme entre le Périgordien ancien et l'Aurignacien, sans convaincre les adversaires de ce modèle qui n'y voient alors qu'une contemporanéité entre la fin du Castelperronien et les débuts de l'Aurignacien.

\section{L'AURIGNACIEN «PRIMITIF» MEDITERRANEEN}

D'autres données, issues du Languedoc cette fois, viennent contribuer à la connaissance de ce premier Aurignacien. F. Bazile (1977) identifie, dans deux séquences d'abris du Languedoc oriental, la Laouza et l'Esquicho-Grapaou, un faciès aurignacien "primitif" à lamelles Dufour, placé géologiquement dans l'interstade würmien. Ces données rejoignent celles déjà connues mais incomplètement publiées de l'abri Mochi à la frontière franco-italienne (Blanc, 1953; Laplace, 1977; Kuhn et Stiner, 1998), et celles des fouilles anciennes de la petite grotte de Bize, révisées par $D$. Sacchi et confirmées par les nouvelles fouilles inédites de A. Tavoso. En Catalogne, près de Gérone,N. Soler (1979) publie les résultats des fouilles de l'Arbreda, avec une séquence aurignacienne présentant à la base une industrie identique à celles trouvées sur la côte languedocienne et provençale mais cette fois-ci datées au début des années 90 par le ${ }^{14} \mathrm{C}$ AMS de 38 à $40000 \mathrm{BP}$. Enfin, en Provence, Onoratini (1986) publie les données de la série aurignacienne de la grotte Rainaude 2, qui présente les mêmes caractéristiques typologiques. De la Catalogne à la Ligurie, dans une région où le Castelperronien n'est pas connu, un faciès aurignacien existe donc, plus ancien que l'Aurignacien I (connu à la Salpétrière), bien que particulièrement mal daté ${ }^{3}$. C'est ce même faciès que Cheynier (1965) avait appelé Mochien, en référence au site éponyme de l'abri Mochi.

\section{A LA RECHERCHE DU PLUS ANCIEN AURIGNACIEN EUROPEEN}

Une découverte très importante, due à J. Hahn (1988), est la séquence de la grotte de Geissenklosterle dans la vallée du Haut-Danube (Jura-Souabe). Sous un niveau de Gravettien ancien à pointes de la Font-Robert et fléchettes, se trouvent

\footnotetext{
3 Je ne crois d'ailleurs pas trop à ces dates très anciennes de l'Arbreda et je verrais plutôt des dates ${ }^{14} \mathrm{C}$ autour de 35000 à $36000 \mathrm{BP}$ pour ce faciès méditerranéen.
} 
deux niveaux aurignaciens dont le plus récent (II), Aurignacien I, est daté autour de 34000 à $31000 \mathrm{BP}$ et dont le plus profond (III), Aurignacien 0, est daté autour de $38000 \mathrm{BP}$. L'étude géologique, réalisée par $\mathrm{H}$. Laville, a confirmé la position dans l'interstade würmien de ce dernier niveau. Des études minutieuses de microstratigraphie et de remontages entre les deux niveaux aurignaciens ont confirmé l'existence et l'individualisation des deux niveaux.

Face à un contexte où se mêlent des anciennes données, à la fiabilité douteuse, des nouvelles données pour lesquelles le sensationnalisme s'incline parfois devant la rigueur scientifique (notamment des dates ${ }^{14} \mathrm{C}$ autour de 40000 $\mathrm{BP}$ ), et un fond de polémique permanente entre écoles, il n'était pas étonnant que peu de candidats se déclarent pour proposer une synthèse de ce premier Aurignacien. C'est ce que J. Kozlowski et $\mathrm{H}$. Delporte me demandèrent de faire pour le colloque sur l'Aurignacien du congrès UISPP de Bratislava en 1991 (Djindjian, 1993).

II fallait pour cela obtenir les inventaires réalisés suivant les deux systèmes typologiques de D. de Sonneville-Bordes et J. Perrot d'une part, de G. Laplace d'autre part, les convertir en un seul système commun par des procédés mathématiques complexes (régression factorielle), et les traiter par analyse des données. Un faciès Aurignacien 0 ou Proto aurignacien émerge effectivement de ces analyses où se côtoient faute de mieux des décomptes à la fiabilité variable mais qui ne perturbent que de façon limitée la stabilité de la structure obtenue. Ce faciès se caractérise par l'importance d'un débitage lamellaire, à l'origine de grattoirs carénés atypiques, différents de ceux de l'Aurignacien II, et qui peuvent être ici qualifiés de nucléus à lamelles. On retrouve également un plus grand nombre de burins de mauvaise facture, qui pourraient aussi être interprétés comme nucléus à lamelles. Les lamelles Dufour sont présentes en nombre variable mais significatif. Grattoirs en bout de lame, grattoirs sur lame retouchée et lames retouchées sont moins nombreux que dans l'Aurignacien I.

\section{LES DONNEES DISPONIBLES POUR L'AURIGNACIEN DU PERIGORD A LA FIN DES ANNEES 80}

A la fin des années 80 , en Périgord, les données plus ou moins disponibles concernent les séquences stratigraphiques d'une dizaine de sites localisés sur un territoire restreint: La Rochette, La Ferrassie, Le Facteur (fouilles Delporte), Pataud (fouilles Movius), Roc de Combe (fouilles Bordes \& Labrot), Caminade Est et Ouest (fouilles Sonneville-Bordes et Mortureux), Le Flageolet I (fouilles Rigaud), Le Trou de la Chèvre (fouilles Arambourou \& Jude), Le Piage (fouilles Champagne). Nulle part, sauf peut-être à Kostienki en Russie, une telle concentration n'est connue qui permette des corrélations suffisantes pour établir un système régional. Ce schéma de l'Aurignacien du Périgord, défini par les moyens statistiques d'H. Delporte $(1984,1991)$ ou par ceux plus sophistiqués de $F$. 
Djindjian (1981, 1985, 1986, 1993a) qui, dans la dernière référence, a intégré les données du Roc de Combe et de Pataud, est le suivant (Djindjian, 1993a, p. 3233):

- Aurignacien I: abondance de la retouche latérale (40\%), présence significative des pièces esquillées, faible représentation des grattoirs aurignaciens $(12 \%)$, rareté des burins $(6 \%)$,

- Aurignacien II ancien: retouche latérale faiblement représentée (10\%), abondance des grattoirs aurignaciens (28\%), abondance des burins $(26 \%)$, dont en particulier, les burins sur troncature $(10 \%)$, les burins dièdres $(9 \%)$, l'apparition des burins busqués $(3,5 \%)$,

- Aurignacien II récent: retouche latérale faiblement représentée (12\%), remplacement des grattoirs aurignaciens par les burins busqués en forte croissance (15\%), développement important des burins $(38 \%)$ dont les burins dièdres et les burins à troncature latéro-transversale $(11 \%)$, les burins sur troncature $(8 \%)$ et les burins busqués $(15 \%)$,

- Aurignacien III: décroissance générale des grattoirs aurignaciens $(8 \%)$ et des burins $(21 \%)$ surtout les burins busqués $(5 \%)$, nouvelle croissance des pièces à retouche latérale $(20 \%)$,

- Aurignacien IV: nouvelle décroissance des pièces à retouche latérale $(7 \%)$, développement des burins carénoïdes: burins carénés, burins à enlèvements dorsaux transverses, burins nucléiformes, formant un continuum avec les grattoirs épais à museau et becs épais à retouche lamellaire. Les burins busqués typiques sont rares. Les grattoirs aurignaciens typiques restent rares $(9 \%)$; les numéros de la liste-type $n .{ }^{\circ} 27$ à 32 où sont généralement classés les burins carénoïdes représentent environ 35 à $46 \%$ des burins de ce niveau.

Ce schéma, à la différence de celui de $H$. Laville (1975), met en contemporanéité les mêmes faciès et les corrèlent avec des éléments climatiques. Aux épisodes froids, correspondent des industries plus laminaires (Aurignacien l et III), tandis qu'aux épisodes tempérés que sont Arcy et Maisières (Aurignacien II et IV), correspondent des industries plus lamellaires (grattoirs et burins aurignaciens). A cette structure cyclique, s'ajoute une structure évolutive linéaire, marquée principalement par une progression dans la technologie de fabrication des burins et la réduction des outils du substrat moustérien. La seule critique explicite émise en son temps, par J. Ph. Rigaud (1993), concerne la représentativité des séries lithiques et principalement celles de La Ferrassie. Cette critique a été discutée et rejetée dans (Djindjian, 1993a). La représentativité des séries en effet est simple à prouver: il s'agit de supprimer dans l'ensemble des 10 séquences, une séquence après l'autre et de vérifier la stabilité des résultats (Djindjian, 1993a), en commençant bien sûr par supprimer la séquence de la Ferrassie. Les résultats sont inchangés. Sans la séquence de La Ferrassie, et en ne conservant même que les 
sites fouillés par l'Institut du Quaternaire, le schéma aurignacien du Périgord reste identique!

Une autre critique, indirectement liée à la question aurignacienne, était la conséquence de la remise en cause des méthodes de reconstitution du cadre paléoenvironnemental des remplissages de grottes et d'abris-sous-roche, notamment l'étude géologique des remplissages, la palynologie (en particulier l'existence des interstades du Würm récent: Arcy, Tursac, Laugerie, Lascaux, etc.) et l'importance des mécanismes post-dépositionnels des couches archéologiques (solifluxion, cryoturbation, lessivage, etc.). Les études quaternaires de pointe, sans lesquelles il n'était point de travail sérieux, devenaient soudainement sulfureuses ! A quoi bon dès lors trouver une structuration de l'Aurignacien corrélée avec des épisodes climatiques, si ces épisodes climatiques étaient eux-mêmes contestés? Certes les critiques méthodologiques avaient des arguments sérieux concernant la fiabilité des enregistrements climatiques des remplissages d'abris-sous-roche: remplissages lacunaires et événementiels, phénomènes de solifluxion et de cryoturbation, dynamique des processus de remplissage mal étudiés, percolation des pollens dans les remplissages, biais par sur- ou sous-représentation des pollens, etc. Face à ce raz de marée dévastateur, comment garder son sang froid? II fallait constater d'abord que les enregistrements climatiques considérés comme fiables par les paléoclimatologues (lacs glaciaires, calotte glaciaire, carotte océanique, sols fossiles des séquences loessiques) présentaient des oscillations de température et d'humidité dans la période $40000-10000$ BP correspondant aux «interstades" déjà décrits, étude que nous avons réalisée il y a quelques années et récemment publiée (Bosselin et Djindjian, 2002). II fallait ensuite revenir sur chaque séquence stratigraphique d'abris-sous-roche et les réinterpréter en termes de dynamique de remplissage (les abris-sous-roche enregistrent bien les épisodes froids), de représentativité botanique du cortège pollinique (et ne plus se contenter d'un pic de pin ubiquiste pour identifier un interstade) et de reconstitution des zoocénoses (à partir des rongeurs mais également des grands mammifères pour les épisodes les plus tempérés: Equus hydruntinus, cerf mégacéros, chevreuil, sanglier, cerf). Dans cette approche plus critique et donc plus rigoureuse, la reconstitution climatique de certaines séquences apparaît douteuse mais certaines autres sont confirmées. II faudra cependant encore quelques années pour que le retour de balancier s'arrête à sa place normale. De cette nouvelle approche, il apparaît que les séquences de La Ferrassie, de Pataud (cette dernière après révision des erreurs d'interprétation chrono-stratigraphique de W. Farrand, cf. Djindjian, 1993) et du Facteur peuvent être considérées comme fournissant une séquence fiable du paléoenvironnement. 


\section{LA MISE EN CAUSE DE L'INTERSTRATIFICATION ENTRE CASTELPERRONIEN ET AURIGNACIEN AU PIAGE ET AU ROC DE COMBE ET LA QUESTION DE L'AURIGNACIEN O EN PERIGORD}

En 1995, à Ravello, je me suis exprimé sur le sujet de l'interstratification entre Castelperronien et Aurignacien au Piage et au Roc de Combe à un colloque international sur la chronologie des industries du Paléolithique supérieur organisé par $F$. Widemann et $Y$. Taborin, et dont les actes viennent enfin d'être publiés (Djindjian, 2003). Ma position à cette époque, en 1995, et je n'ai pas changé d'avis depuis, était de conclure qu'il n'y avait pas d'interstratification entre Castelperronien et Aurignacien dans les sites du Piage et du Roc de Combe ! La révision des industries de la séquence de Roc de Combe (Djindjian, 1993a) m'avait en effet convaincu qu'il n'était pas possible, compte tenu de la dizaine d'outils peu caractéristiques dans une stratigraphie peu sûre à la base, de conclure à l'interstratification d'un niveau aurignacien dans les niveaux castelperroniens. Dans le site du Piage, la situation est plus complexe. II existe en effet plusieurs niveaux aurignaciens $\mathrm{K}, \mathrm{J}$, Gl sous le niveau castelperronien $F 1$, qui vient s'intercaler partiellement au milieu du niveau $F$ aurignacien dans la publication officielle (Champagne et Espitalié, 1981, figures 3, 65 $\& 66$ ). Mais le niveau castelperronien n'est situé que dans un redan du banc rocheux dans la partie nord du site et ne recouvre que très partiellement les niveaux aurignaciens. En outre, les décomptes typologiques de la séquence aurignacienne révèlent une succession atypique, exactement comme si la stratigraphie était inversée ou comme si des remaniements importants étaient survenus ou comme si les fouilles n'avaient pas respecté la stratigraphie naturelle du remplissage (noter également le mélange des industries du niveau CE: Badegoulien et Solutréen). Quelques tentatives inédites de remontage entre couches effectuées sur les séries conservées au Musée des Antiquités Nationales s'étaient d'ailleurs révélées fructueuses*. En attente d'une révision des industries en se basant sur des remontages pour mettre en évidence, carré par carré, l'importance des liaisons entre couches, il semble plus prudent de réserver tout diagnostic sur le point critique d'une interstratification aurignacien-castelperronien, pour laquelle ce site est le dernier candidat, même si la quasi-totalité des spécialistes de l'Aurignacien n'ont pas encore renié l'existence d'une interstratification au Piage et à Roc de Combe: Delporte (1998, p. 88), Demars (1992), Rigaud (1993), etc.

\section{LES APPORTS DU MOUVEMENT TECHNOLOGIQUE A L'AURIGNACIEN DU PERIGORD}

La technologie lithique, relancée sous l'impulsion de $\mathrm{J}$. Tixier au début des années 80 , s'efforce de substituer aux typologies considérées comme des

* Travail entrepris par F. Le Brun-Ricalens, avec l'aide de D. Buisson, et poursuivi par J.-G. Bordes. Pour une synthèse sur la question des interstratifications du Roc-de-Combe et du Piage, cf. Bordes, 2002 (note des éditeurs). 
classifications arbitraires d'objets lithiques mélangeant, entre autres, des outils, des nucléus, des armatures, des pointes et des préhensions diverses, des types technologiques dont la validité est basée sur des remontages, des expérimentations et des études tracéologiques. Dans le cadre de ce nouveau paradigme, les "cultures" paléolithiques ou "faciès culturels" ou "faciès industriels" ou "technocomplexes" suivant les terminologies utilisées, n'auraient que peu de valeur parce que basées sur des structurations effectuées sur des typologies "fourre-tout». Ce serait donc aussi le cas du système aurignacien précédent.

Concernant l'Aurignacien, cependant, plusieurs études technologiques avaient déjà été réalisées bien avant les années 80 . Le débitage laminaire aurignacien avait, depuis le début du $X X^{\circ}$ siècle, déjà été caractérisé comme produisant des grandes lames épaisses et arquées, bien différentes du débitage gravettien, plus fin et rectiligne. Néanmoins, jusqu'à présent, aucune étude quantitative systématique n'a été menée pour discriminer les produits laminaires du débitage de nucléus prismatiques castelperroniens, aurignaciens et gravettiens, afin d'en tirer des règles diagnostiques. Une très bonne étude comparative entre le débitage castelperronien et le débitage aurignacien a été cependant effectuée par $F$. Guilbaud (Guilbaud, thèse non publiée, et 1987) sur les données des fouilles à Quinçay et Saint-Césaire (Levèque, 1987). Les nombreux tests du $X^{2}$, propres aux études laplaciennes, n'ont pas pu révéler malheureusement ici les structures intéressantes. L'analyse des correspondances, que j'ai effectuée sur les données de cette thèse (résultats inédits), met en évidence sur le premier axe une opposition entre les niveaux du site de Quinçay et les niveaux du site de Saint-Césaire, variabilité due aux dimensions et à la nature de la matière première, tandis que le deuxième axe met en opposition les niveaux aurignaciens (de Saint-Césaire) et les niveaux castelperroniens (de Saint-Césaire et de Quinçay), double structure que ne pouvait déceler les simples tests du $X^{2}$. II est donc possible, et c'est sans doute le résultat le plus prometteur de la thèse de $\mathrm{F}$. Guilbaud, de différencier par une analyse quantitative, les débitages aurignaciens et castelperroniens. Cette étude systématique reste également à faire.

Demars \& Laurent (1989) indiquent une différence morphologique entre des lamelles courbes produites par des grattoirs carénés ou des burins carénés ou des burins busqués typiques d'un Aurignacien évolué et des lamelles droites produites par des nucléus à lamelles, typiques d'un Aurignacien 0 . II serait intéressant de généraliser cette étude sur un nombre plus important de niveaux et de sites aurignaciens et castelperroniens par une analyse quantitative plus poussée. $B$. Schmider et M. Perpère (2002), dans l'étude de la couche VII de la grotte du Renne à Arcy-sur-Cure, sont arrivées à une conclusion voisine avec les lamelles Dufour, courtes et torses produites par les nucléus/burins carénés. Au-delà de ces tentatives limitées par la référence typologique de la lamelle Dufour, une problématique plus globale se dégage, celle de l'étude du débitage lamellaire et de ses caractéristiques morpho-techniques dans le Castelperronien, l'Aurignacien et le Gravettien. Cette étude systématique reste également à faire. 
Plus généralement, les grattoirs carénés, les grattoirs à museau épais, les burins busqués et les burins carénés sont considérés par les techno-lithiciens comme des nucléus à lamelles. $H$. Delporte, ces dernières années, avait cependant souligné à plusieurs reprises qu'il fallait distinguer entre les nucléus à lamelles présents dans l'Aurignacien et les vrais grattoirs carénés et burins busqués caractéristiques de l'Aurignacien II. Nous touchons là un point critique dans les propositions des deux parties. Pour $\mathrm{H}$. Delporte, l'abondance des grattoirs carénés et des grattoirs à museau épais correspond à un faciès chronologique dans l'évolution de l'Aurignacien (stade lla), suivi par l'arrivée brève du burin busqué (Ilb) qui n'est qu'une variante technologique du grattoir caréné (enlèvement lamellaire à partir de la face d'éclatement pour le premier et à partir d'un négatif d'enlèvement de coup de burin pour le second). Pour les techno-lithiciens, le grattoir caréné est un nucléus à lamelles, qui devrait se retrouver également à tous les stades de l'Aurignacien. Quand il n'y a pas de grattoirs carénés dans le décompte typologique d'une ancienne fouille, il faudrait donc soupçonner le fouilleur de les avoir pris pour des nucléus (la liste type de D. de Sonneville-Bordes et J. Perrot est d'ailleurs particulièrement floue sur ce point en créant des types tombés en désuétude comme le grattoir nucléiforme et le rabot) et de ne pas les avoir décomptés (voire jetés dans les déblais comme le reste du débitage) ! On peut ainsi imaginer que l'un des buts des nouvelles fouilles de l'abri Castanet pour J. Pelegrin (et R. White) était de trouver les grattoirs carénés du faciès Castanet dans les déblais. C'est sans doute pourquoi également, F. Bon a repris les fouilles de Régismont-le-Haut, faciès typique d'Aurignacien I toujours sans grattoirs carénés. On comprend mieux ainsi pourquoi, «le point de vue technologique» tient tant également à perpétuer l'existence du faciès Lartet-Ferrassie de l'Aurignacien ancien (I), dont nous avons parlé plus haut comme d'un faciès de mélange, puisqu'il contient les grattoirs carénés dus aux mélanges avec l'Aurignacien II sus-jacent des anciennes fouilles ! Que resterait-il en effet des variations diachroniques de l'outillage aurignacien si on retirait de la liste tous les grattoirs et burins aurignaciens parce que considérés comme nucléus? Dès lors que les variations de l'industrie lithique aurignacienne seraient liées à des techniques de débitage (nucléus et leurs produits laminaires et lamellaires caractéristiques), la typologie, ou ce qu'il en resterait, n'interviendrait plus dans les études de différentiation des faciès, qu'ils soient diachroniques ou synchroniques. Ainsi F. Bon (2002), dans sa thèse sur la révision de trois sites aurignaciens pyrénéens: Brassempouy (Landes), Saint-Jean de Verges (Ariège) et Régismont-le-Haut (Aude), distingue les sites suivant leurs modes de débitage: les sites présentant un débitage laminaire sur nucléus prismatique continué en nucléus lamellaire jusqu'à épuisement et les sites présentant à la fois un débitage laminaire sur nucléus prismatique et un débitage lamellaire sur grattoir caréné. II en déduit une classification de l'Aurignacien en deux faciès technologiques, qui ne possèderaient pas de variations diachroniques. S'appuyant, en outre, sur l'étude des sites de La Louza et de l'Esquicho-Grapaou fouillés par $F$. Bazile et étudiés par S. Sicard (Bazile et Sicard, 1999), et sur la révision de la couche VII de la grotte du Renne à Arcy-sur-Cure sous la direction de B. Schmider (2002), il conclut à la 
similarité des modes de débitage, basés sur des nucléus à lames réutilisés en nucléus à lamelles. II en déduit que les trois sites appartiennent à un même faciès Aurignacien 0 , et propose une diffusion de l'Aurignacien 0 (depuis les dates hautes ou basses de 38 à $36000 \mathrm{BP}$ des sites du Languedoc à la date de $31000 \mathrm{BP}$ d'Arcy) vers le nord de la France via la vallée du Rhône puis de la Saône jusqu'à la vallée de la Cure. L'Aurignacien 0 pour $F$. Bon n'est donc plus dès lors, comme son nom ne l'indique pas, un faciès chronologique, mais un faciès technologique et synchronique sans variations ni évolutions dans le temps. L'autre faciès technologique, qui voit cohabiter à la fois un débitage laminaire sur nucléus prismatique et un débitage lamellaire sur grattoir caréné, serait propre à l'Aquitaine. La thèse de $F$. Bon fait donc émerger progressivement et implicitement un modèle synchronique, avec deux territoires aurignaciens: le premier sur le Languedoc, la vallée du Rhône, le bassin de la Saône et de la Seine, le second sur l'Aquitaine. Ce modèle est en opposition totale avec le modèle diachronique que l'on a vu émerger progressivement depuis le début du $X X^{\circ}$ siècle: Lartet, Breuil, Peyrony (en quatre stades), de Sonneville-Bordes (avec l'invention du faciès Castanet et du faciès Lartet-Ferrassie pour l'Aurignacien I), Djindjian (cinq stades) et Delporte (six stades).

Revenons un instant sur une critique implicite faite par les techno-lithiciens aux liste-types: le mélange d'outils et de nucléus (les grattoirs et burins aurignaciens) de la liste typologique fausserait les comparaisons entre les décomptes et, en conséquence, les faciès obtenus par les méthodes statistiques n'auraient aucun sens. En fait, une liste de types n'a jamais été une liste d'outils mais une liste d'artefacts susceptible de révéler des faciès (qui restent à interpréter) dans les assemblages d'ensembles clos. II est méthodologiquement autorisé d'y mettre des outils, des armes, des nucléus, des produits et des déchets de débitage, pourvu que ces artefacts portent une information pertinente en termes de variabilité des assemblages. S'il fallait d'ailleurs se restreindre aux seul outils, dont nous ne connaîtrons jamais la fonction mais seulement les usages révélés par les études tracéologiques, la liste serait un inventaire fortement corrélé aux zones d'activités de l'habitat. A ce stade de la discussion, les techno-lithiciens commettent une première erreur, celle de séparer par exemple des burins-nucléus de burins-outils ou des grattoirs-nucléus de grattoirs-outils puisque les deux technologies de taille sont identiques (obtention par différents moyens d'enlèvements lamellaires multiples). Mais pourquoi des burins à enlèvement uniques ne seraient-ils pas aussi des nucléus comme dans les industries à nombreux burins dièdres: Gravettien final, Magdalénien? Et les pièces esquillées? En réalité, cette distinction est de nature tracéologique et non technologique.

Aussi, dans la problématique qui nous concerne, le point clé n'est pas de savoir si les grattoirs et burins aurignaciens sont des nucléus ou des outils, mais de savoir si le nucléus à lamelles de l'Aurignacien 0 est sur un plan technologique et morphologique différent du grattoir caréné de l'Aurignacien II ! Et, il l'est comme nous l'avions déjà déclaré en 1993 ! Alors, puisqu'une liste-type n'est pas une liste 
d'outils, pourquoi ne pas y avoir inclus des nucléus ou des éléments de débitage? Tout simplement parce que les nucléus prismatiques à un plan de frappe ou à deux plans de frappe opposés sont présents dans toutes les industries du Paléolithique supérieur, et donc ne sont pas des types pertinents pour différencier ces industries! A ce stade de l'argumentation, l'erreur, celle de ne pas avoir compris que c'est la pertinence des artefacts, quelle que soit leur signification a priori (en termes d'information intrinsèque ou extrinsèque) qui est l'enjeu de base de la construction typologique! Que les burins busqués soient des burins ou des nucléus, l'important est qu'à un certain stade chronologique de l'Aurignacien d'Europe occidentale, cette technique ait été utilisée vers $31000 \mathrm{BP}$, durant l'épisode d'Arcy, ni avant, ni après. Et si, par contre, nous rajoutons dans la liste des types, les nucléus prismatiques à lames qui sont présents dans tous les faciès de l'Aurignacien, la structuration de l'Aurignacien ne s'en trouvera pas modifiée.

Mais il serait injuste de considérer que le développement des études technologiques n'ait pas contribué positivement aux études aurignaciennes. Premièrement, les liste-types, surtout celle de D. de Sonneville-Bordes que nous utilisons toujours tous de façon quelque peu hypocrite (sous forme réduite ou modifiée et sans diagramme cumulatif), sont imparfaites dans leur conception: faiblesse de conception sémiotique, manque de systématique, incohérences descriptives, mélanges entre la morphologie, la technologie et le support, besoin inutile d'inventorier tous les artefacts façonnés, séparation inutile entre débitage et outillage, système fermé, liste d'usage régional, etc. II devient indispensable, sous peine de voir les études sur l'industrie lithique perdre leur légitimité scientifique, de construire un nouveau système descriptif des industries lithiques. A ce titre, les études technologiques ont joué un rôle positif, par leur attitude critique concernant la typologie, pour permettre cette reconstruction. Deuxièmement, les études technologiques, ont déjà apporté des données pertinentes et peuvent en apporter d'autres encore, qui peuvent participer à des typologies décrivant les industries lithiques: descriptions technologiques et non typo-morphologiques des "burins" (comme l'étude réalisée pour les burins de l'Aurignacien et du Gravettien de La Ferrassie: Djindjian, 1981), étude des débitages sur éclat au maximum glaciaire (Badegoulien), façonnage par percussion (au percuteur tendre ou dur) et par pression, morpho-technologie des retouches (comme l'étude réalisée par $\mathrm{B}$. Bosselin (1997) sur les lames retouchées de différentes industries du Paléolithique supérieur), détermination des modules de débitage de lames et de lamelles, détermination de nouveaux types technologiques (comme la pièce de la Bertonne (Lenoir, 1987), etc.

Le débat n'est donc pas sur un combat polémique de paradigmes entre typologie et technologie, mais un programme de recherches pour la découverte de nouveaux traits pertinents que permet l'étude des industries lithiques en multipliant l'acquisition d'informations intrinsèques plus complémentaires que concurrentiels comme la morphologie, la technologie, l'usage, la préhension ou l'emmanchement, l'influence de la richesse, de la qualité et de la taille du rognon de matière première, l'accessibilité et les distances d'approvisionnement, etc. 


\section{LES DERNIERS DEVELOPPEMENTS SUR L'AURIGNACIEN DANS LES DEBUTS DES ANNEES 2000}

Dans un article récent, Zilhao et D'Errico (1999), qu'on n'attendait pas s'intéresser de si près à l'Aurignacien, révisent l'ensemble des sites aurignaciens ayant fourni des datations ${ }^{14} \mathrm{C}$ au-delà de $35000 \mathrm{BP}$, et mettent en doute l'ancienneté de l'apparition de l'Aurignacien dans certains sites. La conclusion de leur argumentation est qu'il n'existe pas de vrai Aurignacien plus ancien que $36000 \mathrm{BP}$. Ces conclusions conduisent à raccourcir la fourchette chronologique de l'Aurignacien de 36000 à $29000 \mathrm{BP}$ environ, alors que, selon les opinions des uns ou des autres, l'Aurignacien apparaîtrait dès 43000 BP, 40000 BP ou 38000 BP. Cet argument place l'arrivée de l'Aurignacien en Europe occidentale bien après l'apparition du Castelperronien pour lequel des datations ${ }^{14} \mathrm{C}$ autour de 39-38 000 BP se multiplient depuis quelques années et confirme un précédent article (D'Errico et alii, 1998) qui remettait en cause l'acculturation du Castelperronien par l'Aurignacien, en se basant sur des données cependant bien discutables des objets de parure des niveaux castelperroniens (considérés par certains comme venant des niveaux aurignaciens) de la grotte du Renne à Arcy-sur-Cure. Cet article a eu comme résultat une levée de bouclier des spécialistes de l'Aurignacien, qui se sont sentis attaqués dans les interprétations de leurs propres données de fouilles, et qui se sont expliqués dans un colloque sur l'Aurignacien, organisés d'ailleurs par Zilhao et D'Errico, au XXIV ${ }^{\circ}$ congrès UISPP à Liège en septembre 2001 , et qui a connu comme on pouvait s'en douter un grand succès. Si, à la suite de ce colloque, les conclusions de cet article ont été rejetées dans la généralité de ses conclusions, cet "événement" a eu cependant le mérite de poser la question de la validité des datations ${ }^{14} \mathrm{C}$ au-delà de $35000 \mathrm{BP}$, et des procédures particulières à prendre pour dater de tels sites, notamment la datation systématique de l'industrie osseuse. Et de fait plusieurs sites annoncés comme Aurignacien très ancien, avec des datations proches de $40000 \mathrm{BP}$, ont fait l'objet de critiques fondées (Castillo en Cantabres, Trou Magritte en Belgique), tandis que d'autres ont vu leurs résultats confirmés (Geissenklosterle en Jura-Souabe).

Dans ce contexte, des résultats très importants sont passés relativement inaperçus, ceux des fouilles de l'abri de Labeko Koba en Pays Basque espagnol (Arrizabalaga et Altuna, 2000) et des nouvelles fouilles menées à Isturitz en Pays Basque français (Normand et Turq, 2001). Dans les deux cas, les séquences stratigraphiques découvertes confirment la séquence de Gatzaria: le niveau aurignacien le plus ancien est un niveau à lamelles Dufour, surmonté par une séquence classique comme on la connaît en Périgord. A Isturitz, une date autour de $36000 \mathrm{BP}$, date pour la première fois ce niveau à lamelles Dufour. Ces nouvelles données réhabilitent en fait partiellement l'existence du Protoaurignacien de $\mathrm{G}$. Laplace, à la différence que son extension géographique semble limitée pour l'instant au Pays basque français et espagnol et aux Cantabres (à Cueva Morin notamment, où de nouvelles datations seraient particulièrement bienvenues). Importants également sont les résultats des nouvelles fouilles de Castanet (Pelegrin 
et O'Farrell, 2001) et de Régismont-le-Haut (Bon, ce colloque), qui confirment la rareté de grattoirs carénés avec des datations ${ }^{14} \mathrm{C}$ autour de $34000 \mathrm{BP}$ et donc la réalité de ce faciès Aurignacien I (anciennement faciès Castanet) que nous avons évoqué précédemment.

\section{CONCLUSIONS}

Les débats sur le plus ancien aurignacien ne sont certainement pas clos. II est facile de trouver un Moustérien tardif, en datant un échantillon pollué et un Aurignacien très vieux, en datant des os plutôt que de l'industrie osseuse. Il est plus difficile de mettre en place des méthodes rigoureuses permettant de réunir des arguments convergeant et surs pour la reconstitution d'un schéma de peuplement au paléolithique supérieur ancien en Europe, synchronique et diachronique. Ces méthodes rigoureuses devront permettre l'élaboration d'un cadre paléoclimatique et chronologique indiscutable. Elles impliquent également la mise en œuvre d'un système descriptif de l'industrie lithique renouvelé, intégrant, en les dépassant et en les formalisant à partir des techniques sémiotiques les plus récentes, les acquis typologiques, technologiques et tracéologiques.

Sur la base des données existantes insuffisantes et encore peu fiables, et utilisant des méthodes de formalisation largement insuffisantes et souvent en régression avec celles utilisées dans les années 70 et 80 , il est possible de proposer un schéma encore provisoire pour ce premier peuplement aurignacien. Un Aurignacien initial est connu sur les côtes de méditerranée occidentale de Ligurie jusqu'en Catalogne. Cet Aurignacien est encore mal daté mais pourrait se situer entre 38000 et 35000 BP. L'expansion de cet aurignacien se manifeste également sur les bords septentrionaux des Pyrénées à partir de la côte méditerranéenne jusque dans les Landes, le Pays basque, les Cantabres et les Asturies, sous la forme de ce Protoaurignacien décrit par G. Laplace, qui succède à un Castelperronien ancien, et dont les dates se situeraient entre 36000 et $35000 \mathrm{BP}$. Puis, avec le changement climatique du premier épisode froid du dernier pléniglaciaire, à partir de $34000 \mathrm{BP}$, sous la forme de l'Aurignacien I, l'Aurignacien continue son expansion en Aquitaine avant de faire la conquête de toute les zones septentrionales de l'Europe dans l'épisode tempéré d'Arcy vers $31000 \mathrm{BP}$.

\section{BIBLIOGRAPHIE}

Arrizabalaga, A. \& Altuna, J. (2000): Labeko Koba (Arrasate, Pais Vasco). "Hienas y humanos en los albores del paleolitico superior". Munibe (Antropologia Arkeologia), $n .{ }^{\circ} 52$.

BAZILE, F. (1977): Nouvelles données sur le paléolithique supérieur ancien en Languedoc oriental. $X X^{\circ}$ CPF, Provence, 1974. SPF: 24-28.

BAZILE, F. \& SICARD, S. (2000): Le premier aurignacien du Languedoc oriental dans son contexte méditerranéen. XXIV ${ }^{\circ}$ CPF, Carcassonne 1996. SPF: 117-125.

Blanc, A. C. (1953): // Riparo Mochi ai Balzi Rossi di Grimaldi: Le Industrie. Paleontografic Italica, 50. 
Bon, F.(2002): L'Aurignacien entre mer et océan. Réflexion sur l'unité des phases anciennes de l'Aurignacien dans le Sud de la France. SPF, mémoire $n .{ }^{\circ} 29$.

Bordes, F. (1961): Typologie du Paléolithique ancien et moyen. Bordeaux. Delmas.

Bordes, F. \& SonNeVILLE-Bordes, D. de (1966): Protomagdalénien ou Périgordien VII? L'Anthropologie, t. $70(1-2): 113-122$.

Bordes, F. \& LABROT, J. (1967): La stratigraphie du gisement de Roc-de-Combe et ses implications. B.S.P.F., t. $64(1): 15-28$.

BORDES J.-G. (2002): Les interstratifications Châtelperronien/Aurignacien du Roc-de-Combe et du Piage (Lot, France). Analyse taphonomique des industries lithiques; implications archéologiques. Thèse de doctorat de l'université de Bordeaux I, ex. multigraph., $364 \mathrm{p}$.

Bosselin, B. (1997): Le Protomagdalénien du Blot. Liège: ERAUL, n. 64.

BOSSELIN, B. \& DJINDJIAN, F. (2002): Un essai de reconstitution du climat entre $40000 \mathrm{BP}$ et $10000 \mathrm{BP}$ à partir des séquences polliniques de tourbières et de carottes océaniques et glaciaires à haute résolution. Archeologia E Calcolatori, 13: 275-300.

Breult, H. (1912): Les subdivisions du Paléolithique supérieur et leur signification. XIV ${ }^{\circ}$ Congrès International d'Anthropologie et d'Archéologie préhistorique, Genève: 165-238.

BREZILLON, M. (1971): La dénomination des objets de pierre taillée. XX Supplément à Gallia-Préhistoire. Paris: CNRS.

Bricker, H. M.. \& DAviD, N. C. (1984): Excavation of the abri Pataud, Les Eyzies (Dordogne): The Perigordian VI (level 3) assemblage. American School of Preh. Research, n. ${ }^{\circ} 34,109$ pág., 33 fig.

Campy, M., Chaline J. \& VuILlemey M. (1989): La Baume de Gigny (Jura). XXVIIº supplément à Galliapréhistoire. CNRS

Champagne, F. \& Espitalie, R.(1967): La stratigraphie du Piage. Note préliminaire. B.S.P.F., t. 64 (1): $29-$ 34.

CHEYNIER, A. (1965): Comment vivait l'homme des cavernes à l'âge du renne. Paris. Editions du Scorpion.

COMBier, J. (1951): Gisements paléolithiques de Roclaine, à Romanèche-Thorins (Saône-et-Loire). Revue archéologique de l'Est et du Centre-Est, II, 1:27-39.

Combier, J. \& MonteT-WHite, A. (2002): Solutré (1968-1998). Mémoire n. ${ }^{\circ}$ XXX. Paris. Société Préhistorique Française.

DeLPORTE, H. (1955): L'industrie de Châtelperron et son extension géographique. XIV Congrès CPF, Strasbourg-Metz, 1953. S.P.F.: 233-249.

- (1957): La grotte des Fées de Châtelperron (Allier). XV CPF, Poitiers-Angouleme, 1956. S.P.F.: 452477.

-. (1964): Les niveaux aurignaciens de La Rochette (Dordogne). Bull. Soc. Etudes Recherches Préhist., 13: $52-75$.

- (1968): L'Abri du Facteur à Tursac: I, Etude générale. Gallia-Préhistoire, t. 11-1: 1-112.

- (1984): Le grand Abri de La Ferrassie. Fouilles 1968-73. Paris: Université de Provence, Etudes quaternaires, mémoire n. ${ }^{\circ} 7$.

- (1991): La séquence aurignacienne et périgordienne sur la base des travaux récents réalisés en Périgord. B.S.P.F., t. 88 (8): 243-256.

- (1998): Les Aurignaciens, premiers hommes modernes. La Maison des Roches, 1998, 125p., 51 fig.

Delporte, H., Maziere, G. \& DJINDJIAN, F. (1977): L'Aurignacien de La Ferrassie: observations préliminaires à la suite de fouilles récentes. B.S.P.F., t. 74: 343-361.

DEMARS, P.-Y. (1985): La signification de l'Aurignacien $V$ dans l'évolution des cultures lithiques au Paléolithique supérieur en France. IN Otte (ed.): "La signification culturelle des industries lithiquest" Actes du colloque de Liège, octobre 1984. BAR Intern. Series, n. ${ }^{\circ} 239$, pág. 328-336.

- (1992): L'Aurignacien ancien en Périgord et le problème du Protoaurignacien. Paléo, 4: 101-122.

Demars, P.-Y. \& LauRent, P. (1989): Types d'outils lithiques du Paléolithique supérieur en Europe. Cahiers du Quaternaire, n. ${ }^{\circ} 14$, Bordeaux. CNRS.

DJINDJIAN, F. (1980): Les faciès chronologiques aurignaciens et périgordiens à La Ferrassie (Dordogne). Les Dossiers de l'Archéologie, $\mathrm{n} .^{\circ} 42:$ 70-74.

- (1985): Typologie et culture: l'exemple de l'Aurignacien. IN "La signification culturelle des industries lithiques, M. Otte Edt" Actes du colloque de Liège, octobre 1984. BAR Intern. Series, n. ${ }^{\circ} 239$ : 338 364.

- (1986): Recherches sur l'Aurignacien du Périgord à partir des données nouvelles de La Ferrassie. L'Anthropologie, t. 90, n. ${ }^{\circ}$ 1: 89-106.

- (1993a): L'Aurignacien du Périgord: une révision. Préhistoire européenne, vol 3: 29-54.

- (1993b): Les origines du peuplement aurignacien en Europe. In: "Actes du XII Congrès de l'UISPP. Bratislava 1991", Institut d'Archéologie de l'Acad. Slov. des Sciences, 1993, Vol. 2:136-154. 
- (1999): Chronologie du peuplement gravettien sur les côtes de Méditerranée occidentale. Actes du XXIV ${ }^{\circ}$ Congrès SPF. Carcassonne, Septembre 1994 (France). In Sacchi (ed.) Les Faciès leptolithiques du nord-ouest méditerranéen: Milieux naturels et culturels. SPF, XXIV CPF: 127-138.

- (2003): Chronologie et climato-stratigraphie du Paléolithique supérieur ancien français à partir des données du Périgord. Actes du colloque international de Ravello, 1995. In Widemann \& Taborin (eds.): Chronologies géophysiques et archéologiques du Paléolithique supérieur. Archeologia, storia, cultura n. ${ }^{\circ}$ 3: $283-298$.

DJINDJIAN, F. \& BosSELIN, B. (1994): Périgordien et Gravettien: l'épilogue d'une contradiction? Préhistoire européenne, $n .^{\circ} 6: 117-132$.

DJINDJAAN, F.; KozLowskI, J. \& OTTE M. (1999): Le Paléolithique supérieur en Europe. Paris. Armand Colin.

D'Errico, F.; ZilhaO, J.; Julien, M.; BafFier, D. \& Pelegrin, J. (1998): Neanderthal acculturation in Western Europe? A critical review and its interpretation. Current Anthropology, v.39, supplement: S1S44.

GARROD, D. A. E. (1938): The upper palaeolithic in the light of recent discoveries. Proceedings of the Prehistoric Society, 1: 155-172.

ESCALON DE FonTON, M. (1956): Préhistoire de la Basse-Provence. Etat d'avancement des recherches en 1951. Préhistoire, t. XII, 154 pág., 110 fig.

GuILBAUD, M.(1987): Elaboration d'un cadre morphotechnique pour l'étude du débitage en typologie analytique de quelques industries des gisements de Saint-Césaire (Charente-Maritime) et de Quinçay (Vienne). IN Vandermeersch (ed.): Préhistoire en Poitou-Charentes: problèmes actuels. Paris: CTHS, p. $101-113$.

HAHN, J. (1988): Die Geissenklösterle-Höhle im Achtal bei Blaubeuren I. Landesdenkmalamt BadenWürttemberg, Konrad Theiss, Verlag Stuttgart, 264 pág.

Kunn, S. L. \& Stiner, M. C. (1998): The earliest Aurignacian of Riparo Mochi (Liguria, Italy). Current Antropology, v.39, supl. 3: S175-S189.

LACORRE, F. (1960): La Gravette, le Gravetien et le Bayacien. Laval.

LAPLACE, G. (1966): Recherches sur l'origine et l'évolution des complexes leptolithiques. Paris: De Boccard.

- (1977): II Riparo Mochi ai Balzi Rossi di Grimaldi (Fouilles 1938-1949): Les industries leptolithiques. Rivista Di Scienze Preistoriche, 32: 3-131.

LAVILLE, H. (1975): Climatologie et chronologie du Paléolithique en Périgord. Etude sédimentologique de dépôts en grottes et sous abris. Bordeaux: Institut du Quaternaire, Etudes quaternaires, n. ${ }^{\circ} 4$.

LENOIR, M. (1987): La pièce de La Bertonne, "fossile directeur" du magdalénien ancien? B.S.P.F., t. 84 (6): $167-171$

LERoI-Gourhan, A. (1961): Les fouilles d'Arcy-sur-Cure (Yonne), Gallia-Préhistoire, IV: 3-16.

- (1965): La préhistoire. Paris: PUF.

- (1963): Châtelperronien et Aurignacien dans le Nord-Est de la France (d'après la stratigraphie d'Arcysur-Cure (Yonne). Aurignac et l'Aurignacien, Centenaire des fouilles d'Edouard Lartet. p. 75-84.

LeRoY-Prost, Ch. (1975, 1978): L'industrie osseuse aurignacienne. Essai régional de classification: Poitou, Charentes, Périgord. Gallia Préhistoire, t. 18, fasc.1: 65-156 et t. 22, fasc.1: 205-370.

LEVEQUE, F. (1987): Les gisements castelperroniens de Quincay (Vienne) et de Saint-Césaire (CharenteMaritime): quelques comparaisons préliminaires. Stratigraphie et industries. In Vandermeersch (ed.) Préhistoire de Poitou-Charentes. problèmes actuels. Editions du C.T.H.S.: 91-98.

Movius, H. L.; DAVID, N. C.; BRICKER, H. M. \& CLAY, R. B. (1968): The analysis of certain major classes of upper palaeolithic tools. American School of Prehistoric Research, t. 26, 58p.

NORMAND, Ch. \& TURQ A. (2001): L'Aurignacien de la grotte d'Isturitz: la production lamellaire dans la séquence de la salle Saint-Martin. XIV ${ }^{\circ}$ Congrès UISPP, Liège 2001. Préactes, p. 160.

OnoratinI, G. (1986): Découverte en Provence orientale (grotte Rainaude) d'une industrie souche de l'Aurignacien. Cette civilisation est-elle monolithique? B.S.P.F., t. 83 (8):240-256.

Pelegrin, J. \& O'FarRell, M. (2001):Sur la production lamellaire de l'abri Castanet (Dordogne). XIV ${ }^{\circ}$ Congrès UISPP, Liège 2001. Préactes, p. 161

PeYRony, D. (1933): Les industries aurignaciennes dans le bassin de la Vèzère. B.S.P.F., t. 30: 543-559.

Peyrony, D. (1934): La Ferrassie, Moustérien, Périgordien, Aurignacien. Préhistoire, III, 92p., 89 fig.

- (1948): Le Périgordien, l'Aurignacien et le Solutréen en Eurasie, d'après les dernières fouilles. B.S.P.F., t. 65: 305-328.

Peyrony, D. \& Peyrony, E. (1938): Laugerie-Haute, près des Eyzies, Dordogne. Mémoire Institut Paléontologie Humaine, $n .^{\circ} 19,84 p ., 56$ fig.

PaAdel, L. \& Chollet, A. (1950): L'abri périgordien de Laraux (commune de Lussac-les-Chateaux) (Vienne). L'Anthropologie, t. 54, (3-4): 214-227. 
Pradel, L. (1952): La grotte périgordienne et aurignacienne de Fontenioux (Vienne). B.S.P.F., t. 49: 413432.

- (1959): Le Périgordien II de la grotte des Cottés (Vienne). B.S.P.F., t. 56: 421-427.

Rigaud, J.-Ph. (1993): L'Aurignacien dans le Sud-Ouest de la France: Bilan et perspectives. In: “Actes du XI/ Congrès de I'UISPP-Bratislava 1991", Nitra: Institut d'Archéologie de l'Acad. Slov. des Sciences, Vol. 2: 181-186.

SCHMIDER, B. (2002): L'Aurignacien de la grotte du Renne. Les fouilles d'André Leroi-Gourhan à Arcy-surCure (Yonne). XXXIV' supplément à Gallia-Préhistoire. Paris: CNRS.

SOlER, N. (1979): La secuencin estratigrafica de la cova de l'Arbreda (Serina, Gerona). Actas de la IVe reunion del Grupo de Frabajo del Cuaternario Bañolas: 223-232.

Soler Masferer, N. \& Maroto, J. (1993): Les nouvelles datations de l'Aurignacien dans la péninsule ibérique. In: "Actes du XI/ Congrès de I'UISPP-Bratislava 1991", Nitra: Inst. Archéol. de l'Acad. Slov. des Sciences, 1993, Vol. 2: 162-173.

SONNEVILLE-BORDES, D. DE (1955): La question du Périgordien II. B.S.P.F., t. 52: 197-203.

- (1960): Le Paléolithique supérieur en Périgord. Bordeaux: Delmas, 1960, 558 pág., 295 fig., 64 tabl.

- (1982): L'évolution des industries aurignaciennes. Aurignacien et Gravettien en Europe, Colloque Cracovie-Nitra, 1980. Liège: ERAUL 13, fasc. Il: 339-355.

SONNEVILLE-Bordes, D. DE \& PERROT J. (1953): Essai d'adaptation des méthodes statistiques au Paléolithique supérieur. Premiers résultats. B.S.P.F., t. 50: 322-333.

- (1954-56): Lexique typologique du Paléolithique supérieur. B.S.P.F., t. 51: 327-335; t. 52: 76-79; t. 53: 408-412; t. 53: 547-559.

ZILHAO, J. \& D'ERRICO, F. (1999): The Chronology and Taphonomy of the Earliest Aurignacian and its implications for the understanding of Neandertal extinction. Journal of World Prehistory, 13 (1): 1-68.

ZILHAO, J., AuBRY, Th. \& ALMEIDA, F. (1999): Un modèle technologique pour le passage du Gravettien au Solutréen dans le sud-ouest de l'Europe. Actes du XXIV ${ }^{\circ}$ Congrès CPF. Carcassonne, Septembre 1994 (France). In Sacchij (ed.): Les Faciès leptolithiques du nord-ouest méditerranéen: Milieux nature/s et culturels. Paris: SPF: 165-184. 